\title{
Comparative genomics of Lbx loci reveals conservation of identical Lbx ohnologs in bony vertebrates
}

\author{
Karl R Wotton ${ }^{1}$, Frida K Weierud ${ }^{2}$, Susanne Dietrich ${ }^{* 1}$ and \\ Katharine E Lewis ${ }^{2}$
}

Address: ${ }^{1}$ King's College London, Department of Craniofacial Development, Floor 27 Guy's Tower, Guy's Hospital, London Bridge, London, SE1 9RT, UK and ${ }^{2}$ Cambridge University, Physiology Development \& Neuroscience Department, Anatomy Building, Downing Street, Cambridge, CB2 3DY, UK

Email: Karl R Wotton - kwotton@talk21.com; Frida K Weierud - fkw20@cam.ac.uk; Susanne Dietrich* - susanne.dietrich@kcl.ac.uk; Katharine E Lewis - KEL36@cam.ac.uk

* Corresponding author

Published: 9 June 2008

BMC Evolutionary Biology 2008, 8:17| doi:10.1/86/|47|-2|48-8-|7|
Received: 20 February 2008

Accepted: 9 June 2008

This article is available from: http://www.biomedcentral.com/I47I-2/48/8/171

(C) 2008 Wotton et al; licensee BioMed Central Ltd.

This is an Open Access article distributed under the terms of the Creative Commons Attribution License (http://creativecommons.org/licenses/by/2.0), which permits unrestricted use, distribution, and reproduction in any medium, provided the original work is properly cited.

\begin{abstract}
Background: Lbx/ladybird genes originated as part of the metazoan cluster of $\mathrm{Nk}$ homeobox genes. In all animals investigated so far, both the protostome genes and the vertebrate $L b x I$ genes were found to play crucial roles in neural and muscle development. Recently however, additional $L b x$ genes with divergent expression patterns were discovered in amniotes. Early in the evolution of vertebrates, two rounds of whole genome duplication are thought to have occurred, during which $4 \mathrm{Lbx}$ genes were generated. Which of these genes were maintained in extant vertebrates, and how these genes and their functions evolved, is not known.

Results: Here we searched vertebrate genomes for $L b x$ genes and discovered novel members of this gene family. We also identified signature genes linked to particular Lbx loci and traced the remnants of $4 L b x$ paralogons (two of which retain $L b x$ genes) in amniotes. In teleosts, that have undergone an additional genome duplication, $8 L b x$ paralogons (three of which retain $L b x$ genes) were found. Phylogenetic analyses of $L b x$ and $L b x$-associated genes show that in extant, bony vertebrates only $L b x I$ - and $L b x 2$-type genes are maintained. Of these, some $L b \times 2$ sequences evolved faster and were probably subject to neofunctionalisation, while $L b x I$ genes may have retained more features of the ancestral $L b x$ gene. Genes at $L b x I$ and former $L b x 4$ loci are more closely related, as are genes at $L b \times 2$ and former $L b x 3$ loci. This suggests that during the second vertebrate genome duplication, $L b x / / 4$ and $L b \times 2 / 3$ paralogons were generated from the duplicated $L b x$ loci created during the first duplication event.
\end{abstract}

Conclusion: Our study establishes for the first time the evolutionary history of Lbx genes in bony vertebrates, including the order of gene duplication events, gene loss and phylogenetic relationships. Moreover, we identified genetic hallmarks for each of the $L b x$ paralogons that can be used to trace $L b x$ genes as other vertebrate genomes become available. Significantly, we show that bony vertebrates only retained copies of $L b x I$ and $L b x 2$ genes, with some $L b \times 2$ genes being highly divergent. Thus, we have established a base on which the evolution of Lbx gene function in vertebrate development can be evaluated. 


\section{Background}

Ladybird/Lbx proteins are part of a clustered set of $\mathrm{Nk}$ transcription factors, which encompass the $\mathrm{Nk} 4$ protein tinman/Nkx2, the Nk3 protein bagpipe/Nkx3, ladybird/ Lbx, C15/Tlx, and the Nk1 protein slouch/Nkx1 [1-4]. In protostome genomes, $N K$ genes are organised into a cluster, which, along with the molecular phylogeny of $\mathrm{Nk}$ homeodomains, indicates that they originated via tandem duplications [2]. Indeed, the NK gene cluster is believed to be ancient, with aspects predating the divergence of sponges, cnidarians and bilaterians [5]. In deuterostomes, however, tight clustering has been lost with only Nkx4/ $N k x 3$ and $L b x / T l x$ linkages remaining in the cephalochordate amphioxus and in vertebrates [3].

$L b x$ genes have essential roles in development, including crucial functions in neural and mesodermal cell specification. In mammals and amphibians, $L b \times 1$ controls the emigration of specialised, motile muscle precursors that give rise to limb and hypoglossal/tongue muscles, respectively [6-9]. In mouse embryos, Lbx1 is also required for the specification of several populations of dorsal spinal cord interneurons, which receive and process somatosensory information from the body [10-13]. Moreover, $L b x$ genes with a similar spinal cord expression pattern to mouse have been described in chick, zebrafish and the spotted dogfish and, in the case of zebrafish and chick, these genes are also expressed in muscle [14-16]. Interestingly, in Drosophila melanogaster, a protostome whose ancestors split from the deuterostome lineage over 500 million years ago, two tandem-duplicated ladybird/Lbx family members exist (ladybird early and ladybird late) that are also expressed in developing muscle and neural tissue and are required for the specification of subpopulations of myoblasts and neural cells [1,17]. Thus, $L b x$ genes may have evolutionarily ancient functions in muscle and neural development.

Despite the intriguing similarities of some ladybird/Lbx genes, significant differences become apparent when considering all of the members of the $L b x$ family in different vertebrates. For example, mammals, including humans, have two $L b x$ genes [2] as does the chicken [15,18], whereas Danio rerio has three $L b x$ genes (this report), and the genome sequencing project for Xenopus tropicalis has so far only revealed one $L b x$ gene (this report). In addition, the expression pattern of mammalian $L b x 2$ is distinct from that of $L b \times 1$ ( $L b \times 2$ is expressed in the urogenital system, eye and brain) [19], whereas the two chicken $L b x$ genes and all zebrafish genes are co-expressed in migratory muscle precursors (K. R. Wotton and S. Dietrich, unpublished data). Yet the second chicken $L b x$ gene is not expressed in neural tissues $[15,18]$ while all three of the zebrafish genes are, albeit in distinct regions (K. E. Lewis, unpublished data). Consequently, the evolutionary and functional relationship of ladybird/Lbx genes is not clear.

It is generally believed that at the base of the vertebrate lineage, the entire genome was duplicated twice $(2 \mathrm{R}$ hypothesis; [20]). This was followed by a further genome duplication in the ray-finned fish lineage at the base of the teleost radiation [21-23]. In all chordate relatives of vertebrates investigated so far, such as the urochordates Oikopleura dioica and Ciona intestinalis and the cephalochordate Branchiostoma floridae (amphioxus), only a single lbx gene has been found [3]. It is, therefore, safe to assume that prior to the two rounds of vertebrate genome duplication, only a single $L b x$ gene existed.

One prediction of the $2 \mathrm{R}$ hypothesis is that a $4: 1$ ratio of genes should have been present in an ancestral vertebrate when compared to their invertebrate relatives. However, with gene duplicates carrying redundant functions, many of these four copies (called ohnologs to indicate that they are paralogs that have originated by a process of wholegenome duplication - see $[24,25])$ were lost. Yet, despite considerable interest in $L b x$ gene function in extant vertebrates, orthologies between $L b x$ genes have not been established. Therefore, it is not known whether different vertebrate lineages have lost the same, or different, $L b x$ ohnologs. This raises two alternative hypotheses for the differences that have been observed between vertebrate Lbx genes.

1. Different vertebrate lineages have retained different $L b x$ ohnologs with distinct expression patterns and/or functions

2. The same $L b x$ ohnologs have been maintained in all vertebrates with differences in expression or function being due to more recent neo- and/or sub- functionalisation.

To distinguish between these possibilities, we have used a number of bioinformatics approaches to characterise the organisation of $L b x$ loci in extant osteichthyan vertebrates and to determine the relationship of $L b x$ genes and genes associated with $L b x$ loci. Our studies identified non-NK genes that were acquired by $L b x / T l x$ region(s) prior to, or during, the two rounds of vertebrate genome duplication and that hence, serve as signature genes for these loci. With the help of these signature genes, the remnants of all four $L b x / T l x$ paralogons (eight in teleosts) were identified. Phylogenetic analyses of $L b x, T l x$ and the co-localising non- $N k$ genes revealed that the first round of whole genome duplication in vertebrates created the ancestor of the $L b \times 1 / T l x 1$ and $L b x 4 / T l x 4$ clusters plus the ancestor of the $L b \times 2 / T l \times 2$ and $L b \times 3 / T l \times 3$ clusters. After the second genome duplication, $L b x$ and $T l x$ genes were lost, such 
that before the split of the ray-finned and lobe-finned fish lineages only $L b x 1 / T l x 1, L b x 2 / T l x 2$ and $T l x 3$ genes were maintained. Gene loss also occurred after the additional genome duplication in the ray-finned fish lineage, leaving teleosts (including zebrafish and pufferfish) with two $L b x 1$ and $T l x 3$ genes but only one $L b x 2, T l x 1$ and $T l x 2$ gene. Since the amniote $L b x 2$ genes diverge much more in their coding sequences than the $L b x 1$ genes and the expression pattern of mouse $L b x 2$ is distinct from nonmammalian $L b x 2$ genes, we propose that amniote (or possibly sarcopterygian) $L b \times 2$ genes have evolved at a faster rate and were subject to neofunctionalisation. $L b x 1$ genes on the other hand may have retained more features of the original chordate $l b x$ gene.

\section{Results \\ Identification of Lbx genes in extant Osteichthyes}

To reconstruct the phylogeny of vertebrate $L b x$ genes we first attempted to identify the complete set of these genes in representatives of extant Osteichthyes. For this, BLASTsearches of sequence databases were carried out, using the known human and mouse Lbx1 and Lbx2, chicken Lbx1 and Lbx3, Xenopus laevis Lbx1 and zebrafish Lbx1 sequences as query sequences $[1,2,4,9,14,15,18,19]$. To obtain outgroups for these phylogenetic analyses, we also searched the databases for lbx/ladybird sequences in invertebrate deuterostomes, including the cephalochordate Branchiostoma floridae (amphioxus), the urorchordates Oikopleura dioica and Ciona intestinalis, and the echinoderm Strongylocentrotus purpuratus (purple sea urchin); moreover we included sequences from various protostomes. Our search confirmed the presence of two distinct $L b x$ genes in placental mammals and marsupials (human, mouse, dog, cattle, opossum), one Lbx gene in the still incompletely sequenced platypus and Anole lizard genomes, and two $L b x$ genes in the chicken. Only one $L b x$ gene was found for the frog Xenopus tropicalis. On the other hand, besides the gene so far known as zebrafish $l b x 1$ [14], two novel $l b x$ genes were identified in this organism. Three $L b x$ genes were also identified in the teleosts Takifugu rubripes (fugu), Tetraodon nigroviridis, Gasterosteus aculeatus (stickleback), and two genes in Oryzias latipes (Medaka), while only one lbx/ladybird gene was retrieved for the invertebrate deuterostomes.

\section{Phylogenetic analysis of osteichthyan Lbx protein sequences}

To determine the evolutionary relationship between osteichthyan Lbx genes, we first determined the phylogenetic relationship of Lbx proteins (Fig. 1). For this purpose, amino acid sequences were aligned and analysed, using maximum likelihood methods. We found that the vertebrate Lbx sequences were assigned to two distinct groups. The first group encompassed the known human, mouse, chicken and frog Lbx1 proteins. In addition, this group contained the two novel zebrafish Lbx proteins, encoded by the genes located on chromosomes 13 and 1 , the fugu Lbx proteins whose genes are on scaffolds 52 and 62 , the Tetraodon protein encoded by the $L b x$ gene on chromosome 18, the stickleback proteins with genes on groups VI and IX and the medaka protein encoded by the gene on chromosome 1.

The second group contained all of the mammalian Lbx2 proteins, the chicken protein currently known as Lbx3, the zebrafish protein so far named Lbx1 and encoded by the gene on chromosome 14, together with the Lbx sequences encoded by genes on fugu scaffold 70, Tetraodon chromosome 20, stickleback group IV and medaka scaffold 1066. The division into two distinct groups of Lbx proteins was supported by high bootstrap values. Our phylogenetic analysis shows significantly longer branch lengths for the amniote Lbx2 proteins, indicating that these $L b x 2$ genes have probably evolved at a quicker rate than the $L b x 1$ genes. No evidence was found for distinct Lbx3/4 proteins.

\section{Comparison of genomic Lbx loci in Osteichthyes}

It is generally held that in the lineage leading to jawed vertebrates, two rounds of whole genome duplication occurred, followed by a further genome duplication in the lineage leading to teleosts $[20-23,26]$. Thus, theoretically, if no gene loss has occurred, four Lbx genes should be detectable in extant tetrapods and eight in teleost fish. However, our phylogenetic analyses suggest that only two $L b x$ genes were retained after the second vertebrate genome duplication and before the genome duplication in ray-finned fish. To confirm these results and to further analyse the orthology of the various vertebrate $L b x$ genes, we compared the organisation of genes associated with the vertebrate $L b x$ loci, reasoning that orthologous $L b x$ genes would share a similar chromosomal environment, while paralogous genes would exhibit a distinct arrangement of the locus (see [27-29] for examples).

Since the human genome information is the most accurate and complete, we began by recording the genes that according to the NCBI Map Viewer database are located in the environment of human LBX1 and 2 genes (Fig. 2). Subsequently, we looked for remnants of the LBX3 and 4 loci, initially by searching the human genome for paralogues of the genes co-localising with LBX1 and 2. We then identified and determined the arrangement of genes characteristic for each of these four paralogons in the genomes of other mammals, the chicken, the frog Xenopus tropicalis and the five teleosts (Fig. 3 and additional file 1).

\section{Lbx loci in humans}

Human $L B X 1$ has previously been mapped to chromosome 10, 1.7 Mb distant from NKX2.3 [2,4]. LBX1 is 
Ladybird homeobox $(L b x)$

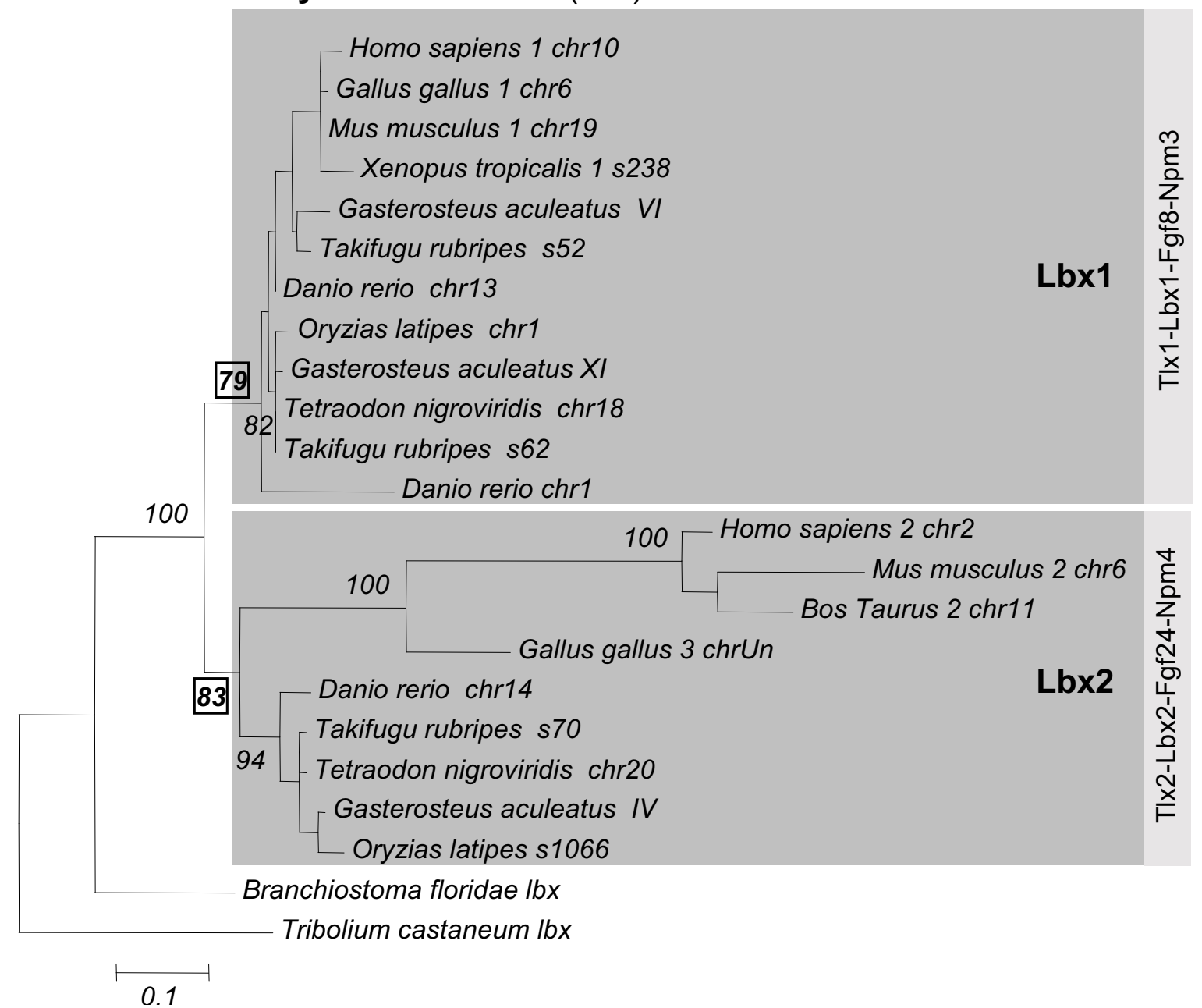

Figure I

Molecular phylogenetic analysis of $L b x$ sequences. The tree shows a maximum likelihood analysis of Lbx protein sequences. Dark grey boxes indicate orthologous groups while light grey boxes indicate gene linkage. Bootstrap values below 70 have been removed. Only two Tetraodon Lbx proteins are included as the third sequence (found on scaffold 8483) is incomplete. Note that the Lbx sequences separate into two clear groups, LbxI and Lbx2, which are supported by bootstrap values of 79 and 83 (boxed), respectively. The chicken protein previously denoted as Lbx3 [18], the zebrafish protein previously denoted as LbxI [14] and one of the fugu, Tetraodon, stickleback and medaka sequences group with mammalian Lbx2 sequences. The two novel zebrafish Lbx sequences and the remaining teleost sequences group with mammalian, chicken and frog Lbxl. The tree shows significantly longer branch lengths for the Lbx2 proteins indicating that these sequences may be evolving at a quicker rate than the LbxI proteins. For common names of species see additional file 2.

tightly linked to the related Nk-type homeobox gene TLX1 (Fig. 2 and Additional file 1). This is followed by the Kazal-type serine peptidase inhibitor domain 1 gene KAZALD1, the LOXL4 gene encoding a Lysyl oxidase like protein and the SLIT1 gene encoding an axon guidance molecule. Moving in the opposite direction, LBX1 is linked to a Beta-transducin repeat containing gene (BTRC1, a F-box and WD repeat domain 11 type gene), the DNA polymerase lambda gene $(P O L L)$, the Deleted in a mouse model of Primary Ciliary Dyskinesia gene
(DPCD), the F-box and WD repeat domain containing 4 gene (FBXW4), the Fibroblast growth factor 8 gene (FGF8), the Nucleophosmin 3 gene (NPM3), the Meningioma expressed antigen 5 gene (MGEA5), the gene encoding a $\mathrm{Kv}$ channel interacting protein 2 (KCNIP2), and further away, the gene encoding the Lim domain binding protein LDB1 and the NKX1.2 gene (Figs $2 \& 3$ ).

Human $L B X 2$ has been mapped to chromosome 2, and is linked to TLX2 ([2,4]; Fig. 2). Neither of these genes are 


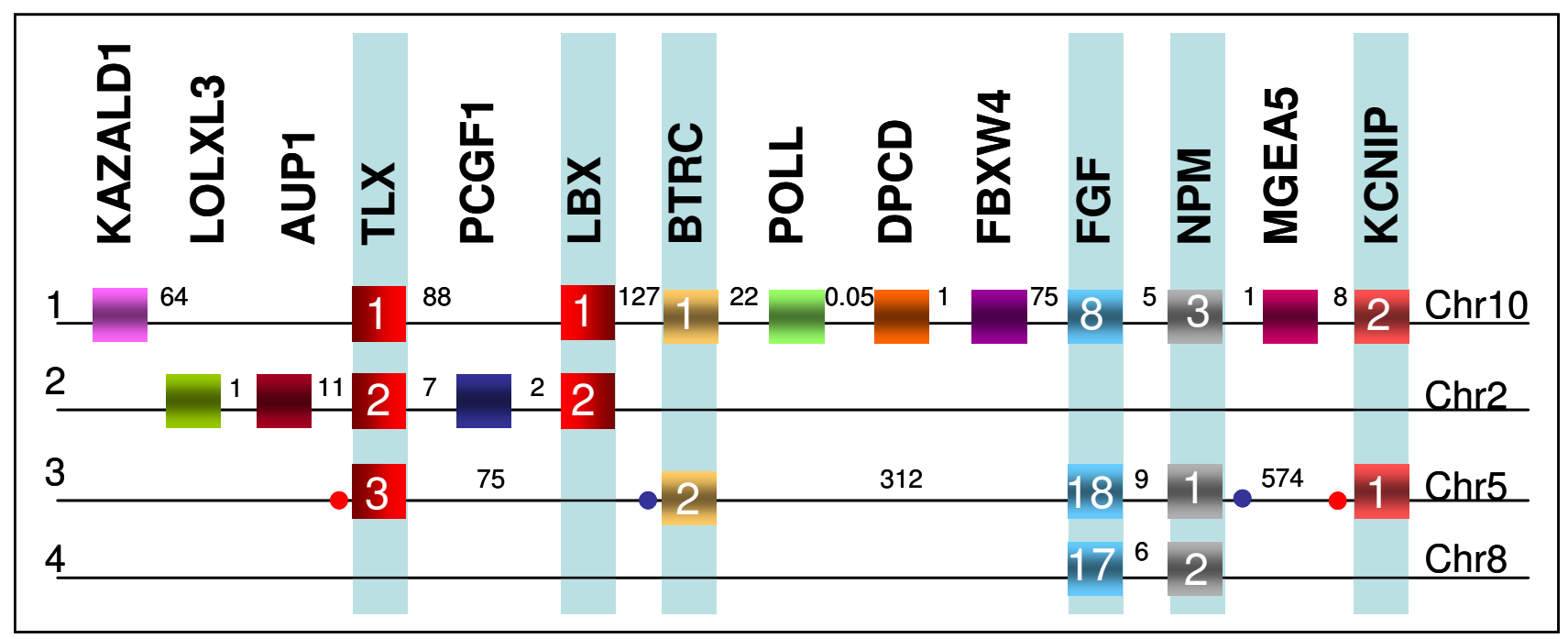

Figure 2

Human paralogons of the LBXITLX cluster loci. Schematic representation of the four human $L B X / T L X$ paralogons plus paralogous genes occurring at the NKX3.2 locus, deduced from analysis of the human genome using NCBI map viewer Homo sapiens Build 36.2 (September 2006). Genes are represented by boxes with gene names appearing above and gene subfamilies indicated by numbers inside. Letters on the far left indicate paralogon designation, while chromosomal locations are indicated on the far right. Numbers between genes are approximate intergenic distances in Kb. Background shading indicates paralogous genes. Blue and red dots indicate sites of inversions. Note that LOXLI, AUPI and PCGFI genes appear only at the LBX2 locus and KAZALDI, POLL, DPCD, FBXW4 and MGEA5 appear only at the LBXI locus. BTRC, FGF, NPM and KCNIP genes are linked with $L B X$ and $T L X$ genes at more than one locus, suggesting that these genes were acquired by the $L B X / T L X$ cluster during or before the two rounds of vertebrate genome duplication. Also note that a KCNIP4 and a SLIT2 gene are associated with NKX3.2 on chromosome 4. A KCNIP3 gene is located on the long arm of chromosome2, however at a distance to LBX2, suggesting that it is not an original component of the $L B X 2$ paralogon.

associated with other $N K$ genes, suggesting that they have translocated from their original cluster [2,3]. LBX2 and TLX2 are separated by the PCGF1 gene, which encodes a polycomb group ring finger. Facing away from PCGF1 and TLX2, LBX2 is linked to genes that encode Dynactin subunit 1 (DCTN1) and the Tetratricopeptide repeat protein 31 (TTC31). TLX2 on the other side is flanked by the DEAQ box 1 (DQX1) gene which encodes for an ATPdependent RNA helicase, the gene encoding Ancient ubiquitous protein 1 (AUP1), the HTRA1 gene which encodes for a high temperature requiring serine protease, the LOXL3 gene, DOK1 which encodes for the Docking downstream of Tyrosine kinase 1 protein, and a gene encoding the uncharacterised protein NP620159.2. Thus, the human LBX1 and LBX2 loci are quite distinct (Fig. 2). However, they both harbour TLX genes, in line with the idea that $L b x$ and $T l x$ genes were linked in the original bilaterian cluster of Nk-type homebox genes [2,3]. Moreover, both $L B X$ loci include LOXL genes.

In humans, a further TLX gene exists $1.4 \mathrm{Mb}$ distant from NKX2.5 on chromosome 5, thought to be a remnant of the third cluster of $N K$ genes, which, after the second ver- tebrate genome duplication, lost its cognate $L B X$ gene ([3]; Fig. 2, Additional file 1). Investigating the organisation of this former $L B X 3 / T L X 3$ locus, we found that TLX3 is on one side linked to the gene that encodes for Ranbinding protein 17 (RANBP17, a member of the Exportin protein family), the gene encoding for the gamma-aminobutyric acid receptor (GABRP), a further KCNIP gene, KCNIP1, the Lymphocyte cytosolic protein gene LCP2, the Forkhead transcription factor gene FOXI1, the Dedicator of cytokinesis gene DOCK2, the coil-coil domain encoding CCDC99 gene, followed by SLIT3. On the other side, TLX3 is linked to NMP1, FGF18, FBXW11/BTRC2, and further away from the TLX3 gene, NKX2.5, MSX2 and DOK3. The order of NMP1, FGF18 and FBXW11/BTRC2 is reversed compared to the paralogous genes at the $L B X 1 /$ TLX1 locus. However, it is remarkable that for both the $L B X 1 / T L X 1$ and $L B X 3 / T L X 3$ paralogons, the NPM and FGF genes are closely linked, separated by sequences of only 5 and $9 \mathrm{~kb}$, respectively. Taken together, our data suggests that Kcnip, Fbxw11/Btrc2, Slit, Loxl, Dok and the closely associated Fgf-Npm genes are hallmarks of $L b x / T l x$ loci. 


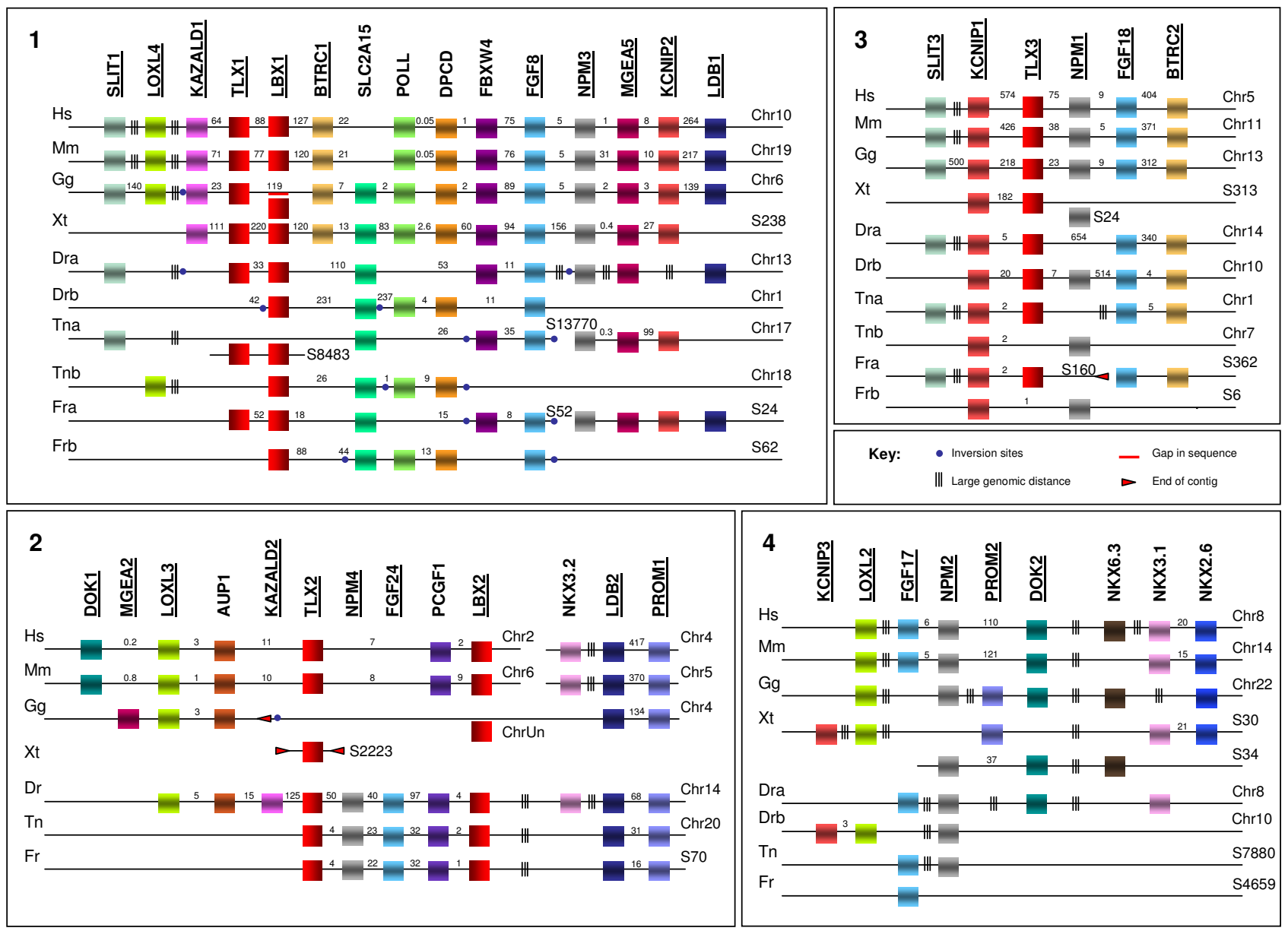

\section{Figure 3}

Genomic organisation of human LBX/TLX cluster paralogons and putative orthologous counterparts. Genomic organisation of human (Hs) Lbx/Tlx paralogons and the putative orthologous counterparts identified in Mus musculus (Mm), Xenopus tropicalis (Xt), Gallus gallus (Gg), Danio rerio (Dr), Tetraodon nigroviridis (Tn) and Takifugu rubripes (Fr). For simplicity, only three teleosts are included in this figure: additional data for Oryzias latipes (medaka) and Gasterosteus aculeatus (stickleback) can be found in additional file I. The main genes characteristic of each paralogon are included in the figure; additional genes and linked genes found further away can be found in additional file I. Schematic representations of the orthologous genomic regions are depicted in panels I-4, which correspond to human paralogons I-4 in figure 2. Gene orthology is indicated by colour code and was inferred from molecular phylogenetics (see additional files). Genes appearing in more than one orthologous region are underlined. Numbers at the ends of each line indicate chromosome (chr) or scaffold (s) numbers. A parallel red line marks gaps in the sequence, while triple black lines indicate large intergenic distances. Blue dots mark sites of inversions, red arrows the end of a contig. Boxes off-line represent genes for which genomic sequences are incomplete; where no chromosome or scaffold number is given, no linkage data is available. In some cases, "missing" genes can be found on different scaffolds or chromosomes. This data is shown in additional file $I$. In addition, we do not show the dispersed $2^{\text {nd }}$ teleost Lbx2 paralogon that no longer contains an Lbx or Tlx gene, but this information is included in additional file $I$. The genes our phylogenetic analysis identified as $L b x \mid$ are found in a similar genomic environment, linked to orthologs of the genes present at the human LBXI/TLXI locus. In addition, non-mammalian LbxI genes are linked to the Slc2al5 gene not found at any other Lbx locus. Lbx2 genes are linked to Loxl3, Aupl and PcgfI genes (genomic information incomplete for chicken and frog), while TIx3 genes are linked to the orthologs of human KCNIPI, NMPI, FGFI8 and BTRC2. In teleosts, Fgf24 and Npm4 are part of the Lbx2 locus, while in all species examined, FgfI 7 and $\mathrm{Npm} 2$ are no longer linked to $L b x / T / x$ genes.

To identify remnants of the fourth $L B X$ locus, we searched the human genome for additional, linked KCNIP, BTRC/ FBXW11, SLIT, LOXL, DOK and FGF-NPM sequences. No further homologues of BTRC/FBXW11 genes were identified. However, we found:

- FGF17 and NPM2 closely linked to DOK2 and about 1.8 Mb distant from LOXL2, NKX2.6 and NKX3.1 on chromosome 8, which also carries NKX6.3
- Linked KCNIP4-SLIT2 genes $7.7 \mathrm{Mb}$ distant from NKX3.2 on chromosome 4

- KCNIP3 on the long arm of chromosome 2, however $20.9 \mathrm{Mb}$ distant from and hence probably not a genuine part of the LBX2-TLX2 region.

A previous report suggested that $L B X 2 / T L X 2$ may once have belonged to the NKX2.6-NKX3.1 region on chromosome 8 [3]. However, the presence of LOXL and DOK 
genes at both the LBX2 locus and the NKX2.6-NKX3 locus is not consistent with this idea. KCNIP4-SLIT2 were found linked to the Prominin gene PROM1, so far not associated with $L B X / T L X$ loci. However, as KCNIP3 is also linked to PROM2, this suggests that Prom genes may once have belonged to $L b x / T l x$ containing regions. Taken together, these observations suggest that the LBX2/TLX2 region may have originated from the NKX3.2 containing cluster on chromosome 4, while FGF17-NPM2 linked to LOXL2 and $D O K 2$ represent the remnants of the fourth $L B X / T L X$ region associated with $N K X 2.6$ and 3.1 on chromosome 8 .

\section{Lbx loci in other mammals}

Investigating the arrangement of genes at $L b x$ loci in additional placental mammals (mouse, dog and cattle), a marsupial (opossum) and a monotreme (platypus), we found that the loci are arranged in the same fashion as in humans (Fig. 3, Additional file 1 and data not shown). The exception is the platypus $L b \times 2 / T l \times 2$ locus, whose existence could not be confirmed as genes linked to this paralogon in other mammals were found on short, unlinked and poorly characterised DNA fragments. Moreover, in mouse and Monodelphis, Prom2-Kcnip3 are not on the same chromosome as the Lbx2 locus, supporting the idea that these genes secondarily intercalated into the $L b \times 2$ carrying chromosome in the lineage leading to humans.

\section{Lbx loci in the chicken}

As the information on the chicken genome is still fragmentary, the localisation of chicken $L b x$ genes could not be determined with certainty. However, chicken chromosome 6 contains a region that is syntenic with the LBX1 region on Human chromosome 10, encompassing Kazald1, Tlx1, Btrc, Poll, Dpcd, Fbxw4, Fgf8, Nmp3, Mgea5 and Kcnip2 in the same order as the human genes (Fig. 3). Loxl4, Slit1 and further genes located in the wider environment of the mammalian $L b x 1$ locus were also identified, with gene groups displaying a similar arrangement in the chicken and in mammals (Additional file 1). Furthermore, we identified sequences 300 bp upstream and 2.2 $\mathrm{kb}$ downstream of the two $L b \times 1$ exons, which are conserved between the mammalian $L b x 1$ loci and the putative chicken $L b x 1$ site; in the chicken these regions flank a region that has not been fully sequenced yet (data not shown). Thus, it is likely that this area on chromosome 6 indeed harbours the $L b \times 1$ gene. As will become relevant below, in the chicken the Btrc1 gene is separated from the Poll gene by the Slc2a15 gene (our name) encoding a solute carrier (Fig. 3).

The Lbx2-type gene so far named $L b x 3$ [18] is situated on a short contig not assigned to a chromosome (Fig. 3). However, Aup1, Htra2, Loxl3, the gene encoding NP620159.2, together with a further Mgea gene, were found on the so far poorly-characterised contig 242 assigned to chromosome 4; all genes are arranged in the same order as the corresponding genes at the Lbx2/Tlx2 containing region of mammalian chromosomes (Fig. 3; Additional file 1). Moreover, separated by sequence gaps, these genes are linked to the Dctn1 gene, which is downstream of $L b \times 2$ in mammals. Significantly, this chromosome also carries genes associated with the NKX3.2 locus in humans, adding weight to the hypothesis that $L b \times 2$ genes were once associated with this particular NK cluster.

Tlx3 was found on chicken chromosome 13 in a region syntenic with the TLX3 containing region of human chromosome 5 and grouped with Fbxw11/Btrc2, Fgf18, Npm1, Ranbp17, Gabrp, Kcnip1, Foxi1, Lcp2, Dock2, Ccdc99 and Slit3 in the same fashion as mammalian Tlx3. In agreement with this being the third $L b x / T l x$ paralogon, other members of the NK cluster associated with this paralogon are also found on chick chromosome 13 (Additional file 1).

Finally, a representative of Fgf17 could not be identified, but Npm2, Dok2 and Loxl2 were found on chromosome 22 , in a region syntenic to the FGF17-NPM2 region on human chromosome 8 and linked to Nkx2.8 and 6.3 (Fig. 3 and Additional file 1). Notably, this region is also linked to Prom2, a gene in mammals associated with Kcnip3 (Kcnip3 was not found in the chicken). This finding supports the idea that Kcnip3-Prom 2 are not an original component of the $L b \times 2 / T l x 2$ paralagon but instead probably belong to the fourth $L b x / T l x$ paralagon.

\section{Lbx loci in Xenopus tropicalis}

In the frog, both Lbx1 and the Kazald1, Tlx1, Btrc, Poll, Dpcd, Fbxw4, Fgf8, Nmp3, Mgea5 and Kcnip2 genes typical for the amniote $L b x 1$ locus were found on scaffold 238 (Fig. 3 and Additional file 1). As in the chicken, the Slc2a15 gene was located between Btrc and Poll, suggesting that the solute carrier gene was present in the original $L b x 1$ locus and was lost from this location in the lineage leading to mammals.

A Tlx2 gene was found on the scaffold 2223, but this scaffold does not contain any further genes associated with $L b x / T l x$ paralagons (Fig. 3 and Additional files). Similarly, an isolated Pcgf1 gene was found on scaffold 47. Searches for Loxl3 and Aup genes were unsuccessful. Thus, the existence of a $L b \times 2 / T l x 2$ locus could not be determined. However, linked Tlx3, Gabrp, Kcnip1, Foxi, Dock2 and Ccdc99 genes were found on scaffold 313 , suggesting that a $L b \times 3 /$ Tlx3 locus exists in frogs (Fig. 3 ).

As in chicken, we did not find a Xenopus tropicalis ortholog of Fgf17, but we detected Npm2 and Dok2 on scaffold 34, linked to each other and arranged in the same order as the 
corresponding genes surrounding human and chicken Npm2. Notably, as in amniotes, these genes are also linked to Nkx6.3 (Additional file 1). Moreover, scaffold 30 contains linked Loxl2, Nkx3.1, Nkx2.6, Prom2 and Kcnip3 genes, which in amniotes, with the exception of Prom2Kcnip3 that have translocated to a different chromosome in placental mammals, are linked to Fgf17, Npm2, Dok2 and Nkx6.3 (Additional file 1). Taken together, this suggests that all of these genes belong to the same Nkx3.1, 2.6 and 6.3 containing cluster.

\section{Lbx loci in teleost fish}

For teleosts, we expected to find chromosomal arrangements corresponding to that of tetrapods, if, in line with our analysis of $L b x$ proteins, only two $L b x$ bearing loci were present prior to the genome duplication in the rayfinned fish lineage. Alternatively, if in teleosts $L b \times 3$ and $L b \times 4$ genes were maintained, they should be embedded in distinct loci, with $L b x 3$ next to Tlx3, Kcnip1, Npm1, Fgf18 and Btrc2 and/or Lbx4 near to Fgf17 and Npm2.

We found for the two novel Lbx1-type teleost genes that the organisation of their gene loci fell into two classes. The genes on zebrafish chromosome 13, Tetraodon scaffolds 8483 and 13770, fugu scaffold 52 and stickleback group VI are surrounded by $T l x 1$ (sequence incomplete for Tetraodon), Slc2a15, Fbxw4 and Fgf8, thus bearing the hallmarks of the tetrapod Lbx1/Tlx1 locus (Fig. 3 and additional files). The same arrangement of genes was found on medaka chromosome 15 , with a sequence gap at the place where the $l b x$ gene is expected to be. Significantly, in all of these teleosts the order of genes is identical to that of the equivalent tetrapod genes, with the exception of an inversion between the Tlx 1 and Fgf8 region (Figure 3). The $L b x$ genes on zebrafish chromosome 1, Tetraodon chromosome 18 (sequence incomplete), fugu scaffold 62, stickleback group IX and medaka chromosome 1 are linked to a second Slc2a15 gene, and to Poll, Dpcd and a second Fgf8type gene (there is a sequence gap at the position of the Fgf8 gene in Tetraodon), thus also bearing hallmarks of the tetrapod $L b x 1 / T l x 1$ locus (Fig. 3 and additional files). Both stickleback and medaka loci are accompanied by Kcnip2 and Mgea5 genes; for stickleback group VI and medaka chromosome 15, these genes are also associated with Npm3 and Slit1, while linked Npm3-Mgea5 genes were found on zebrafish chromosome 13. Moreover, all of the teleost $L b x 1$-type loci are associated with gene groups found in the wider environment of $L b x 1$ loci in amniotes (Additional file 1). Taken together, these findings support the idea that all of these fish have two $L b \times 1 / T l x 1$ paralogons, although in many cases there has been reciprocal gene loss following the teleost-specific whole genome duplication
For the third of the teleost $L b x$ genes, namely the gene currently known as zebrafish $L b x 1$ on chromosome 14 and the Lbx genes on Tetraodon chromosome 20, fugu scaffold 70 , stickleback group IV and medaka scaffold 1066, we found an environment reminiscent of the mammalian $L b x 2$ locus, with the $L b x$ genes being separated from a $T l x$ gene by Pcgf1 (Fig. 3). The exception is the medaka scaffold 1066, which is a short fragment that only contains the $L b x$ and Pcgf genes. Different from that of mammals, an Fgf24-Npm4 gene set was found between the $L b x$ and $T l x$ genes. In the wider environment of the Lbx-Pcgf1Fgf24-Npm4-Tlx gene set, we found two types of arrangement (Additional file 1). On zebrafish chromosome 14, the genes are associated with Aup1 and Loxl3, reminiscent of the mammalian Lbx2/Tlx2 paralagon. The genes on fugu scaffold 70, Tetraodon chromosome 20 and stickleback group IV, are associated with Nanos1, Limch1, Phox2b, Tmem33, Bbs7, Anxa5, Fgfbp, Prom1, Tapt1, Ldb2 (Lbx1 side) and Adad1, Spata5, Ankrd50, Leprot1, Srp72, Arl9, Hop, Sec24B (Tlx side); the ultracontigs 115 and 117 and chromosome 10 of medaka harbour the same genes in the same order. Notably, the genes surrounding the $L b x-P c g f-F g f-N p m$-Tlx group in fugu, Tetraodon, stickleback and medaka are also found on zebrafish chromsome 14, while Aup1 is found on stickleback group IV and medaka chromosome 10 (Additional file 1), i.e. all of these genes were located in the wider environment of the Lbx-Pcgf-FgfNpm-Tlx locus. Taken together, our data suggest that, despite substantial gene rearrangements, these chromosomal regions in teleosts are $L b \times 2 / T l \times 2$ paralogons. Interestingly, the genes closely associated with fugu, Tetraodon, stickleback and medaka Lbx2, including the Fgfbp-Prom1Tapt1-Ldb2-Anxa5 genes, are linked with the Nkx3.2 locus on human chromosome 4 and chicken chromosome 4, consistent with our proposal that the mammalian $L b \times 2$ genes were originally part of the Nkx3.2 cluster (additional file 1).

Searching for remnants of the second teleost $L b x 2$ paralagon, we found Loxl3, a further Nanos1 gene and Ttc31 on Tetraodon chromosome 10, fugu scaffold 338, stickleback group XV and medaka chromosome 22. Dok1NP620159.2-Sema4f genes were found on Tetraodon scaffold 7074, fugu scaffold 186, stickleback group VII and medaka chromosome 18; in stickleback and medaka these genes were also linked to Kcnip4-Gba3-Gpr125, which in tetrapods co-localise with $N k x 3.2$ (Additional file 1; the scaffolds were too short to determine whether this linkage also exists for fugu and Tetraodon). Finally, at a distance from the second $L b x 1$ locus on zebrafish chromosome 1, Tetraodon chromosome 18, fugu scaffold 17, stickleback group IX and medaka chromosome 1, we found Slit2 and a second group of Fgfbp-Prom1-Tapt1-Anxa5 genes linked to $F b x w 7$, which in humans are all associated with the $L B X 2$ locus. Thus, it seems that the second teleost $L b \times 2$ 
paralagon has dispersed, with parts having translocated to one of the Lbx1 carrying chromosomes.

For Tlx3 genes, two types of arrangements were identified (Additional file 1). On zebrafish chromosome 14, fugu scaffold 160, Tetraodon chromosome 1, stickleback group IV and medaka chromosome 10, i.e. inserted into the $L b x 2$ containing chromosome, Tlx3 is on one side linked to Kcnip1, and on the other linked to the Hrh2 gene encoding a Histamine receptor, Dock2 and, slightly more distant, Fgf18 and Fbxw11. On zebrafish chromosome 10, fugu scaffold 6, Tetraodon chromosome 7, stickleback group VII and medaka chromosome 14, Hrh2 is linked with Kncip1, Npm1 and in the case of zebrafish, Tlx $3 b, F \& f 18$ and Fbxw11, suggesting that remnants of the two Tlx3 loci created by the teleost-specific genome duplication still exist.

Finally, searching for additional Btrc, Prom, Loxl, Slit, Dok, Kcnip, and Fgf-Npm genes as indicators of other possible $L b x / T l x$ paralogons, we identified linked Npm2-Dok2F\&f17 genes on zebrafish chromosome 8, linked F\&f17Loxl2-Kcnip3 genes on stickleback group XIII, and linked Kcnip3-Loxl2-Dok2 genes on Tetraodon chromosome 12 and medaka chromosome 9. A second set of linked Kcnip3-Loxl2-Dok2 genes was identified on zebrafish chromosome 10 and a second set of Loxl2 and Kcnip3 genes in a conserved environment but split between two chromosomes were also identified for the other four fish species. Notably, additional genes were also identified that colocalise with Loxl2, Dok2, Kcnip3, Fgf17 and Npm2 genes both in teleosts and tetrapods, including orthologs of Nkx2.6 (Additional file 1 and data not shown), supporting the idea that all these genes once belonged to the same locus, which in tetrapods still encompasses $N k x$ 2.6, 3.1 and 6.3 .

\section{Phylogenetic relationship of signature genes for Lbx/Tlx paralogons}

Characterising $L b x / T l x$ loci in tetrapods, we found evidence for three distinct paralogons, with the possibility of Fgf17-Npm2 representing the remnant of the fourth. In teleosts, the arrangement of $L b x 1 / T l x 1$ and $T l x 3$ genes closely follows the pattern observed in tetrapods. However, the putative teleost $L b \times 2 / T l x 2$ paralogon (genes currently named $L b \times 1$ and $T l x 3 a$ in zebrafish) includes additional Fgf-Npm genes. Moreover, all of the teleosts except zebrafish show a somewhat divergent organisation of genes in the chromosomal regions surrounding the Lbx2/ Tlx2 loci. Furthermore, the duplicate $L b \times 2$ paralogon and the two Fgf17-Npm2 paralogons are poorly preserved in teleosts. Therefore, to further confirm the evolutionary relationship of the Lbx loci and our assignment of genes to specific paralogons, we carried out a comprehensive phylogenetic analysis of protein sequences encoded by the genes in the various $L b x / T l x$ regions. For this, we BLAST- searched sequence databases for related sequences and built phylogenetic trees which included various invertebrate outgroups (Additional file 2).

\section{Phylogenetic relationship of genes co-localising with several Lbx paralogons}

Btrc/Fbxw11, Mgea, Kazald, $L d b$ and Prom genes occurred at two, Tlx, Loxl, Slit and Dok genes at three and Kcnip, and the linked Fgf-Npm genes at four putative Lbx loci in tetrapods. If our assignment of $L b x / T l x$ paralogons is correct, then the genes that we have assigned to particular paralogons should group together. In addition, if the fundamental lay-out of $L b x / T l x$ paralogons was established in vertebrates before the divergence of lobe-finned and rayfinned fish and the additional genome duplication in the ray-finned fish lineage, then the teleost protein sequences should group with the tetrapod proteins. If, however, distinct $L b x / T l x$ loci were maintained in the lineage leading to lobe-finned fish/tetrapods and ray-finned fish/teleosts, then our phylogenetic analysis should reveal additional groups of genes.

With the exception of Dok2 sequences, which seem to have become highly diverged, all of our analyses supported our assignment of the different $L b x / T l x$ paralogons (discussed in detail in Additional file 2). Notably, our analyses of $L d b$ and Prom 1 genes also support our model that the tetrapod $L b \times 2$ loci was originally located in the $N k \times 3.2$ containing cluster: the $L d b 2$ sequences, which are associated with $L b x 2$ in teleosts and with the now $L b x$-less locus carrying Nkx3.2 in tetrapods group together, as do the Prom 1 genes which are linked to Ldb2-Tapt-Anxa5 genes and hence, to the current plus the dispersed $L b x 2$ and Nkx3.2 loci (Fig 3). In addition, our analyses of Prom2, Tlx, Kcnip, Fgf and Npn genes shows that orthologues associated with the $L b x 1$ and $L b x 4$ paralogons are more closely related to each other than to orthologues associated with the $L b x 2$ and $L b x 3$ paralogons and vice versa. This is most informative for the genes where four paralogues are still linked to potential Lbx loci in extant vertebrates (Fgf, Npm and Kcnip genes). In these cases, we found that Kcnip1 and Kcnip4 genes are more closely related to each other than to Kcnip2 and 3. Similarly, Fgf8 sequences are closely related to Fgf17 sequences, and Fof2 4 sequences are closely related to Fgf18. Finally, the Npm3-Npm2 and Npm4-Npm1 sequences are more closely related to each other, respectively (Additional file 2 ). Taken together, this suggests that the $L b x 1 / 4$ paralogons and the $L b \times 2 / 3$ paralagons arose from different ancestral $L b x$ loci generated during the first vertebrate genome duplication.

\section{Discussion}

Ladybird/Lbx genes are crucial regulators of metazoan neural and muscle cell specification [6-9]. Yet before this 
study, it was difficult to evaluate the basic role of $L b x$ genes in vertebrate development and evolution, as it was unclear exactly how many $L b x$ genes exist in different vertebrates and how these genes are phylogenetically related to each other. The $2 \mathrm{R}$ hypothesis predicts that early in the vertebrate lineage, four ohnologs were generated for each gene in the genome; for teleosts, descendants of ray-finned fish, a further genome duplication should have produced eight gene copies [3,20-23,26,30-38]. However four ohnologs are seldom found in vertebrate genomes, suggesting that gene loss has played an important part in genome evolution. In recent years, two $L b x$ genes were identified in amniotes, one in frogs and one in the teleost Danio rerio $[1,2,9,14,15,18,19]$. One of the amniote $L b x$ genes and the $L b x$ genes identified in frog and zebrafish had been reported to be $L b x 1$ genes, while the second of the amniote $L b x$ genes had been suggested to represent distinct $L b \times 2$ (mammals) and $L b \times 3$ genes (birds) with rather divergent expression and function $[18,19]$. This suggested that $L b x 1,2$ and 3 genes were retained in amniotes up to the split of mammalian and avian lineages, while despite their additional genome duplication ray-finned fish seemed to have experienced a near-complete extinction of $L b x$ genes. However, previous $L b x$ gene assignments were sometimes based on limited sequence comparisons, and neither a comprehensive phylogenetic analysis nor a comparative genomic analysis of vertebrate $L b x$ genes had been carried out. Therefore, it remained unclear whether more $L b x$ genes had yet to be found and whether these genes were survivors of $L b x 1,2,3$ or 4 genes.

In this study, we searched vertebrate sequence databases for $L b x$ genes and determined their phylogenetic relationships. Moreover, we investigated the organisation of $L b x$ gene loci, identified signature genes linked to these loci and established the phylogenetic relationships of these genes. Our study confirms the existence of two $L b x$ genes in amniotes, while only one $L b x$ gene was found, thus far, in Xenopus tropicalis. However, we were able to detect novel $L b x$ genes in teleosts. Yet, we found no species with the predicted set of four (lobe-finned fish lineage) or eight (ray-finned fish lineage) $L b x$ genes. Thus, in all bony vertebrates, some $L b x$ genes have been lost. Nevertheless, tracing the signature genes linked to different $L b x$ loci, we were able to identify remnants of all four $L b x$ paralogons in tetrapods and all eight $L b x$ paralogons in teleosts. However, only $L b x 1$ and $L b \times 2$-type loci retained $L b x$ genes; no evidence for surviving $L b x 3$ or $L b x 4$ genes was found. With cognate tetrapod and fish paralogons displaying a high degree of similarity, this suggests that $L b \times 3$ and 4 genes were lost before the split of the lobe-finned and rayfinned fish lineages. Finally, our phylogenetic and synteny analyses revealed that $L b x$-associated genes in $L b x 1-L b x 4$ paralogons and in $L b x 2-L b x 3$ paralogons, respectively, are more closely related. This suggests that $L b x 1$ and $L b x 4$ par- alogons originated from one, and $L b \times 2$ and $L b \times 3$ paralogons from the other, parental paralogon generated during the first round of vertebrate genome duplication.

\section{Two novel Lbx genes were identified in teleosts}

Our searches of genomic databases identified two novel $L b x$ genes in zebrafish, and three Lbx genes in Takifugu rubripes (fugu), Tetraodon nigroviridis and Gasterosteus aculeatus (stickleback). Oryzias latipes (medaka) carries two $L b x$ genes, with a sequence gap at the expected position of the third. Taken together, this suggests that teleosts share the same set of $3 L b x$ genes.

Phylogenetic analyses grouped the two novel zebrafish Lbx proteins, two of the three fugu, Tetraodon and stickleback proteins and one of the medaka proteins with the tetrapod Lbx1 sequences. Conversely, the zebrafish protein currently known as Lbx1, the remaining fugu, Tetraodon, stickleback and medaka sequences and the chicken protein so far called Lbx3 all grouped with the mammalian Lbx2 sequences. The bipartite separation of Lbx sequences was supported by high bootstrap values, suggesting that in all extant Osteichthyes, only $L b \times 1$ and $L b \times 2$ type genes were maintained, with teleosts harbouring duplicates of $L b x 1$.

\section{Traces of four Lbx loci were identified in tetrapods}

Prior to the two rounds of vertebrate genome duplication, the $N K$ clusters broke up and non-NK genes were acquired by these loci ([3] and this study). Our analysis shows that tetrapod Lbx1 is invariantly linked to Tlx1, Kazald1, Btrc, Slc2a15, Poll, Dpcd, Fbxw4, Fgf8, Npm3, Mgea5, Kcnip2, $L d b$, Loxl4 and Slit1 with Slc2a15 being lost in mammals. The assignment of $L b \times 2$-type genes was more problematic, due to incomplete sequence information for chicken and frog. For mammals however, $L b x 2$ was found linked to Tlx2, Pcof1, Aup1, Loxl3 and Dok1, and linked Loxl and Aup genes were also identified for chicken (distribution of signature genes for Lbx loci shown in Table 1).

The linking of $L b x$ with $T l x$ genes was not unexpected, given its maintenance in the otherwise fragmented amphioxus $n k$ cluster [3]. Yet tetrapods harbour a third $T l x$ gene, suggesting that a third, previously $L b \times 3$-containing paralogon still exists [39-41]. Indeed, tetrapod Tlx3 sequences form a phylogenetic group distinct from Tlx1 and Tlx2 sequences, supporting the idea that this gene did not arise from a single gene duplication event. Moreover, Tlx3 loci share the same organisation amongst tetrapods and encompass Btrc2, Fgf18, Npm1, Kcnip1, and Slit3, which according to our phylogenetic analysis are paralogues of the genes found at the Lbx1 site. Thus, while $L b \times 3$ itself was lost from the Tlx3 locus, a number of genes that must have been acquired by the $N k$ cluster prior to 
Table I: Distribution of paralogous genes at the four Lbx-Tlx loci.

\begin{tabular}{|c|c|c|c|c|c|c|}
\hline & LbxI/TIxI locus & Former Lbx4/Tlx4 locus & $\begin{array}{l}\text { (Former Lbx3)/Tlx3 } \\
\text { locus }\end{array}$ & Lbx2/TIx2 locus & Lbx-less Nkx3.2 locus & Lbx2/TIx2 loci \\
\hline & & & & amniotes & tetrapods & teleosts \\
\hline Msx & & & Msx2 & & & \\
\hline $\mathrm{Nk} \times 2 / \mathrm{tin}$ & $\mathrm{Nk} \times 2.3$ & $\mathrm{Nk} \times 2.6$ & $\mathrm{Nkx2.5}$ & & & \\
\hline Nkx3/bap & & $\begin{array}{l}\text { Nkx3.I } \\
\text { (chicken: Nkx2.8 only) }\end{array}$ & & & Nkx3.2 & $\mathrm{Nk} \times 3.2$ \\
\hline Nkx6 & & $\mathrm{Nk} \times 6.3$ & & & & \\
\hline Lbx & LbxI & & & Lbx2 & & Lbx2 \\
\hline Tlx & Tlxl & & TIx3 & TIx2 & & Tlx2 \\
\hline Pol & Poll & Polb & & & & \\
\hline Dpcd & Dpcd & & & & & \\
\hline Fbxw4 & Fbxw4 & & & & & \\
\hline Fbxw7 & & & & & Fbxw7 & Fbxw7 \\
\hline Slc2a 15 & Slc2al 5 & & & & Slc2a9 (tetrapods) & \\
\hline Aup & & & & Aupl & & Aupl \\
\hline Pcgfl & & & & Pcgfl & & Pcgfl \\
\hline Fbxw I I/Btrc & Btrc & & Fbxwl I/Btrc2 & & & \\
\hline Mgea & Mgea5 & & & Mgea2 (chicken) & & Mgea2 \\
\hline Kazald & KazaldI (tetrapods) & Kazald3 (fish) & & Kazald2 (zebrafish) & & \\
\hline Ldb & Ldb I & & & & Ldb2 & Ldb2 \\
\hline Prom & Prom3 (fish) & Prom2 (tetrapods)* & & & Prom I & Prom I \\
\hline Loxl & Loxl4 & Loxl2 & & LoxI3 & & LoxI3 \\
\hline Slit & Slit I & & Slit3 & & Slit2 & Slit2 \\
\hline Dok & & Dok2 & Dok3 & Dokl & & Dokl \\
\hline Fgf & Fgf8 & Fgfl 7 & Fgf 18 & & & Fgf24 \\
\hline $\mathrm{Npm}$ & Npm3 & Npm2 & Npm I & & & Npm4 \\
\hline Kcnip & Kcnip2 & Kcnip3* & Kenip I & & Kcnip4 & Kcnip4 \\
\hline
\end{tabular}

* in human, cattle, dog, Prom2-Kcnip3 are on the same chromosome but at a distance to $L b x 2$.

Note, genes occurring at single $L b x / T l x$ loci form monophyletic trees, i.e. the loci in fish and tetrapods are related (see additional file 2). Genes at more than one locus: gene phylogeny matches genomic localisation and environment (exception: Dok2 genes which are distinct from Dok I but do not group well; see additional file 2). Genes at the putative Lbx4-Tlx4 locus, characterised amongst others by Fgfl 7-Npm2, match between fish and tetrapods. Genes found associated with $L b \times 2$ loci in fish match genes in amniotes distributed between the $L b \times 2$ locus and the $L b x$-free $N k x 3.2$ locus, suggesting that the amniote $L b x 2$ locus once belonged to the Nkx3.2 containing Nk cluster. Genes in bold: genes that supported by high bootstrap values are more related to each other than to other paralogues, suggesting that the $L b x I$ and $L b x 4$ loci evolved from one and the $L b x 2$ and $L b x 3$ from the other of the paralogons generated during the first round of vertebrate genome duplication.

the two rounds of vertebrate genome duplication are still present.

Using the previously identified signature genes linked to the $L b x / T l x$ loci as query sequences, we also identified remnants of the fourth $L b x$ paralogon. Interestingly, both the $L b x 1 / T l x 1$ and the Tlx3 locus contain closely linked $\mathrm{Fgf-Npm}$ genes, and an additional pair of tightly linked Fgf-Npm genes, namely Fgf17 and Npm2, was found in mammals, linked to Dok2, Loxl2, Nkx3.1 and 2.6. Linked Loxl2-Dok2-Npm2 sequences were also found in the chicken genome, and in the frog, linked Dok2-Npm2 genes and Loxl2-Nkx3.1-Nkx2.6 genes were found on two, possibly neighbouring scaffolds. In some tetrapods the Loxl2Nkx3.1-Nkx2.6 genes were also linked to Prom2 and Kcnip3. This suggests that Prom2-Kcnip3 initially belonged to the fourth $L b x$ paralogon that contains Fgf17-Npm2 as well as Dok2-Loxl2-Nkx3.1-Nkx2.6 genes, while the placement of Prom2-Kcnip3 on the same chromosome but at a distance to Lbx2 in humans, cattle and dog (but not mouse) is probably a result of a more recent transposition event. In summary, presence of a number of signature genes for $L b x$ loci linked to $N k \times 2.6$ and $N k \times 3.1$ suggests that this region is the ancestral home of the lost $L b \times 4-T l x 4$ genes.

\section{Duplicates of the four tetrapod-type Lbx loci were identified in teleosts, with two LbxI genes and one Lbx2 gene still present}

In teleosts, the two novel, putative $L b x 1$ genes were found in two types of genomic settings, both of which contained signature genes for the tetrapod $L b x 1$ locus. Genes that occurred at only one of the novel teleost $L b x$ sites formed monophyletic groups with their tetrapod counterparts. Importantly, Slc2a15 and $F g f 8$ genes, which were found at both of the teleost loci as well as the tetrapod Lbx1 sites, also formed single groups, indicating that the two novel $L b x$ loci in teleosts arose from a common $L b x 1$-containing ancestor. Consequently, tetrapod $L b x 1$ genes and the two novel teleost $L b x$ genes are orthologs. 
Similar to the Lbx1 paralogons, the relationship of the now $L b x$-less $T l x 3$ loci could readily be established. Teleosts harbour one locus containing Slit3, Kcnip1, Tlx3, Fgf18 and Btrc2 genes in the same order as found at the tetrapod Tlx3 site, with the exception of Tetraodon whose Slit3 gene intercalated between Kcnip1 and Fgf18. Notably, this Tlx3 locus is found on the same chromosome, but with different integration sites, as the gene we propose to rename $L b x 2$, suggesting extensive transposition between $L b x / T l x$ loci in teleosts. The second $T l x 3$ locus contains Kcnip1-Npm1 and in zebrafish also Tlx3b, plus a second copy of Fgf18 and Btrc2. Further shared genes were found in the environment of the tetrapod and teleost Tlx loci; genes at these sites, including the Tlx genes themselves, grouped together in our phylogenetic analyses. This suggests that teleost $T 7 \times 3$ and $T 7 \times 3 b$ are orthologs of the tetrapod $T l x 3$ genes. The high degree of locus conservation also suggests that the basic lay out of this Tlx3 paralogon, including the elimination of $L b x 3$, was established prior to the teleost-specific genome duplication.

In the zebrafish, the gene currently known as $l b x 1$ but placed with mammalian $L b x 2$ in our phylogenetic analyses was found in a genomic environment bearing the hallmarks of the mammalian $L b \times 2$ locus, being linked with Pcgf1, a Tlx gene currently named Tlx3a, Aup1 and Loxl3. These genes formed monophyletic groups with the cognate genes at the mammalian $L b x 2$ locus, with teleost Tlx3a grouping with mammalian Tlx2. This suggests that this locus is indeed a $L b x 2$ paralogon. In fugu, Tetraodon, stickleback and medaka, the third $L b x$ gene was also linked to Pcgf1 and a Tlx2-type gene, although Aup was located at a distance or in case of Tetraodon, on a different chromosome. The latter seems to be a result of a secondary rearrangement of the locus, as aup is linked to $l b x$ in amphioxus and hence, was part of $l b x$ loci before the split of cephalochordate-vertebrate lineages (aup and $l b x$ are both found on Scaffold 294 of the Branchiostoma floridae v1.0 genome at the JGI. See Availability and requirements section for URL). Nevertheless, all of the teleost $L b \times 2$ loci encompassed a Fgf24-Npm4 set between Pcgf1 and Tlx2. Moreover, genes associated with Prom 1 and directly linked to fugu, Tetraodon, stickleback and medaka $L b \times 2$ were found in the same order in the wider environment of the zebrafish $L b x$-Pcgf1-Tlx set. Phylogenetic analyses indicated that these genes are orthologs, suggesting that the teleost $L b \times 2$ loci are variations on the same theme.

In fugu, Tetraodon, stickleback and medaka, the Lbx2 gene was directly adjacent to Nanos1, which is not linked to the mammalian $L b x 2$ gene. In mammals on the other side, the Lbx2-Pcgf1-Tlx2 set was flanked by Ttc31 and Loxl3-Dok1Np620159.2-Sema4f. Yet in these four teleosts, a second Nanos1 gene was identified, linked to Loxl3, Dok1, Np620159.2, Sema4f and Ttc31. Moreover, a duplicate of the Prom1-linked gene set was found at the same site. A similar arrangement of genes was also detected in the zebrafish, distributed however between two chromosomes and with individual genes missing from the set. Phylogenetic analyses grouped the genes with the cognate genes at the mammalian and teleost $L b x 2$-bearing sites, suggesting that this location is the second, now $L b \times 2 / T l \times 2$ less $L b \times 2$ paralogon in teleosts. As will become relevant below, this locus encompassed Slit2, Kcnip4 and Fbxw7, associated with the $L b x$-free $N k x 3.2$ locus in tetrapods.

Establishing whether teleosts carry duplicates of the possible fourth $L b x$ paralogon was more problematic as unfortunately, some of the genes suggestive of this paralogon (namely Fgf17, Npm2, Dok2, Loxl2, Prom2, Kcnip3) reside on rather small scaffolds and hence, linkage could not always be established. However, all five teleosts harboured two sets of Loxl2-Kcnip3 genes. One of the sets was associated with Dok2 and in stickleback, also with Fgf17. Moreover, Npm2-Dok2-Fgf17 were found together on zebrafish chromosome 8. Phylogenetic analyses grouped Fgf17, Npm2, Loxl2, Kcnip3 genes with their namesakes from tetrapod genomes. This suggests that, although less well preserved, two copies of the putative fourth $L b x$ paralogon still exist in extant teleosts.

\section{The mammalian Lbx2 locus has translocated from the NK cluster containing Nkx3.2}

Previous studies of human $N k$ genes showed that remnants of two $N k$ clusters have been preserved, with chromosome 10 carrying $L B X 1$ and TLX1 linked to NKX2.3 and NKX1.2, and chromosome 5 carrying TLX3 linked to NKX2.5 [3]. More partial remnants of two further Nk clusters have also been identified, one on chromosome 8 with NKX2.6 and NKX3.1 and one on chromosome 4 with NKX3.2 and NKX1.1. However, no LBX or TLX genes are linked to these regions.

A previous study, based primarily on the human genome, proposed that the Nkx2.6, 3.1 and 6.3 paralogon (located on human chromosome 8) might be the ancestral location of $L b \times 2$ and Tlx2 (now located on human chromosome 2) [3]. By a process of elimination, this would further suggest that $L b x 4$ and $T l x 4$ were lost from the NKX3.2 paralogon (currently located on human chromosome 4). Consistent with this hypothesis, paralogs of a few genes (Kcnip, Prom, Add, Adra2) are represented at both the Lbx2 and the Nk3.2 loci in mammals, suggesting that these two loci have derived from separate Lbx/Tlx paralogons. However, phylogenetic analysis of these gene families shows that the mammalian genes linked to the Lbx2 loci group with frog, chicken and/or teleost genes associated with Fgf17 and Npm2 loci (see phylogenetic analysis and discussion in Additional file 2). This suggests that these genes may have translocated to the mammalian 
$L b x 2$ loci from the putative $4^{\text {th }} L b x / T l x$ paralogon (e.g. the genes now on human chromosome 2 may have originally been linked to the $T l x / L b x$ paralogon now on human chromosome 8).

Consistent with this second interpretation our data suggests that the ancestral location of the LBX2/TLX2 paralogon now located on human chromosome 2 was the NKX3.2 paralogon on human chromosome 4 (as shown in Fig. 3). The evidence for this is several fold. Firstly, we have identified four distinct $\mathrm{Fg} f$ and Npm genes, with Fgf8Npm3 demarcating Lbx1/Tlx1 loci, Fgf18-Npm1 the Tlx3 loci, F\&f24-Npm4 the preserved teleost Lbx2/Tlx2 locus and Fof17-Npm2 genes associated with tetrapod and teleost Nkx2.6-3.1-6.3 genes. Secondly, paralogous Loxl and Dok genes are present at both the Lbx2 locus and the Nkx2.63.1-6.3 locus, suggesting that these two loci have evolved from different paralogons. Thirdly, amniote orthologs of genes associated with the two teleost $L b \times 2$ loci such as Fbxw7, Ldb2, Prom1, Slit1 and Kcnip4 co-localise with Nkx3.2, and these amniote and teleost genes fall into the same phylogenetic groups. Fourthly, the Lbx2 locus is still associated with $N k \times 3.2$ genes in teleosts, and possibly, also in the chicken (the signature genes linked to the $L b x 2$ locus are assigned to chromosome 4, but their position is not determined). All together, this strongly suggests that the mammalian Lbx2-Tlx2 locus was once located in the $N k$ cluster containing Nkx3.2 and that $L b \times 4$ and $T l x 4$ were lost from the Nkx2.6-3.1-6.3 paralogon that contains $f g f 17$ and $n p m 2$.

\section{Lbx4-TIx4 were lost prior to the third genome duplication in ray-finned fish}

If the assignment of Lbx2-Tlx2 to the Nkx3.2 carrying Nk cluster is correct then, as discussed above, the $N k$ cluster containing Nkx2.6, 3.1 and 6.3 (and $f g f 17$ and $n p m 2$ ) is the former location of the Lbx4-Tlx4 paralogon. This idea is supported by the presence of a number of signature genes for $L b x$ loci at this site. Moreover, genes at this locus do not group with paralogues accompanying other $L b x$ loci; most notably, the Fgf-Npm and Kcnip genes located here constitute distinct phylogenetic groups. Taken together, this suggests that different to Lbx2-Tlx2 genes, Lbx4-Tlx4 genes did not translocate to another chromosome. Rather, they were lost from this fourth NK cluster. Significantly, both teleosts and tetrapods retained a similar set of genes from this locus. This indicates that the basic lay-out of the paralogon, including the elimination of its $L b x$ and $T l x$ genes, was probably established in an ancestor of the Osteichthyes (i.e. prior to the split of the lobe-finned and ray-finned fish lineages).

\section{Lbx loci in extant vertebrates arose from Lbx I/4 and Lbx 2/3 precursors}

Our study shows that of the genes associated with $L b x$-Tlx loci, Fgf, Npm and Kcnip genes still exist in four copies, inferring that indeed, the original chordate locus was duplicated twice in the lineage leading to vertebrates. Phylogenetic analyses indicated that $F g f 8$ genes found in the $L b \times 1 / T l x 1$ paralogon and Fgf17, found in the Lbx4/Tlx4 paralogon, are more closely related than $F \& f 24$ found in the intact teleost $L b x 2 / T l x 2$ paralogon and Fgf18 found in the $L b \times 3 / T l \times 3$ paralogon. Similarly, Npm4 (teleost Lbx2/ $T l \times 2$ paralogon) and $N p m 1$ ( $L b \times 3 / T l \times 3$ paralogon) are closely related to each other, distinguished from Npm1 (Tlx3 paralogon) and Npm4 (teleost Lbx2 paralogon), A similar grouping was found for Kcnip genes, with Kcnip1 (Tlx3 paralogon) and Kcnip4 (teleost Lbx2 and tetrapod Nkx3.2 loci) being the most closely related. This pair-wise grouping is supported by high bootstrap values and suggests that during the second vertebrate genome duplication, $L b x 1$ and $L b x 4$ paralogons arose from one of the $L b x$ $T l x$ loci generated by the initial duplication event and $L b x 2$ and $L b \times 3$ paralogons arose from the other duplicated locus. Consistent with this model, Tlx2 and Tlx 3 genes are rather similar and are easily distinguished from the Tlx1 genes associated with $L b x 1$.

\section{Basic roles of Lbx I genes and neofunctionalisation of mammalian Lbx2 genes}

Our analysis shows that the organisation of orthologous $L b x / T l x$ paralogons is very similar in extant bony vertebrates, suggesting that the main compositions of the paralogons were established before the divergence of lobefinned and ray-finned fish. This infers that Osteichthyes had only two types of $L b x$ genes. Expression analyses and functional studies on $L b x 1$ type genes suggest that they all play a role in dorsal spinal cord and muscle development [6-16]. Interestingly, the zebrafish and chicken $L b \times 2$ genes are still expressed in muscle precursors, and zebrafish $l b x 2$ is also expressed in the spinal cord [14,18]. In contrast, mouse $L b \times 2$ is not expressed in these regions, rather labelling the urogenital system, eye and brain [19]. Amniote Lbx2 proteins also show the most divergent sequences. This suggests that amniote (or potentially sarcopterygian) Lbx2 genes have evolved at a faster rate than their anamniote (or non-sarcopterygian) orthologs, and undergone neofunctionalisation. Other $L b x 2$ genes, however, may have retained aspects of ancestral $L b x$ expression and function, which seems to be tied to the formation of specific neural and muscle cell types.

\section{Conclusion}

We have identified remnants of all four $L b x / T l x$ paralogons in extant bony vertebrates. Phylogenetic analyses of $L b x, T l x$ and non-NK genes at these loci revealed that the first round of whole genome duplication in vertebrates 


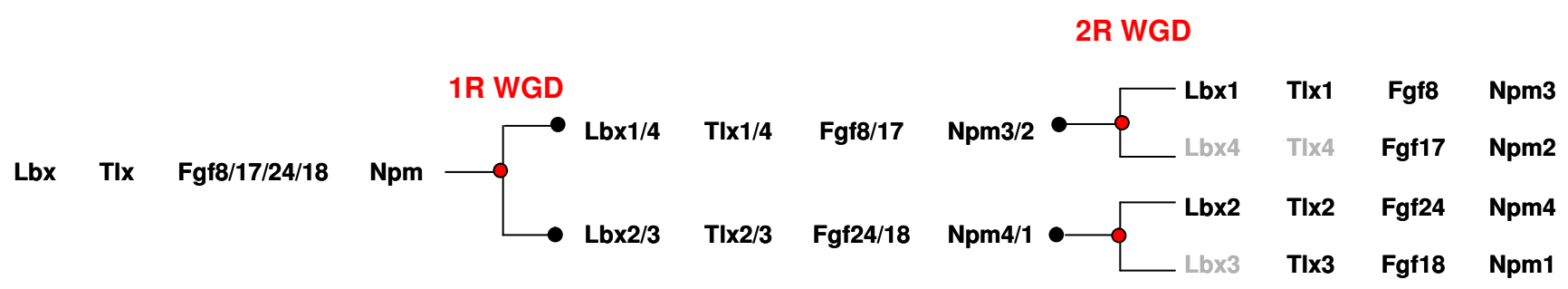

Figure 4

A model of the evolution of the vertebrate Lbx/TIx loci. Lbx loci in extant vertebrates arose from a region containing an Lbx, Tlx, Fgf8// 7/24//8 and an Npm gene. After one round of whole genome duplication (IR WGD) Lbx I/4 and Lbx2/3 precursors were produced linked to Fgf8/I 7 - Npm2/3 and Fgf24//8 - Npm4/I precursors, respectively. During the second round of whole genome duplication (2R WGD), four loci where produced. By the time of the divergence of the lobed-fined and rayfinned fish, the $L b x 4, T l x 4$ and $L b x 3$ genes (shown in grey) had been lost.

created the ancestor of the $L b \times 1 / T l \times 1$ and $L b \times 4 / T l \times 4$ paralogons and the ancestor of the Lbx2/Tlx2 and Lbx3/Tlx3 paralogons (Fig. 4). After the second genome duplication, $L b x$ and $T l x$ genes were lost, such that before the split of the ray-finned fish/teleost and lobe-finned fish/tetrapod lineages only $L b x 1 / T l x 1, L b x 2 / T l x 2$ and $T l x 3$ genes were maintained. In the ray-finned fish lineage, genome duplication and subsequent gene loss left teleosts with two $L b x 1$, one $L b x 2$ and two $T l x 3$ loci. Since the amniote $L b x 2$ genes show divergent amino acid sequences and expression patterns, we propose that these $L b x 2$ genes have been evolving at a faster rate and were subject to neofunctionalisation. $L b x 1$ genes on the other hand may have retained more features of the original chordate $l b x$ gene.

\section{Methods}

Regions surrounding the human LBX/TLX cluster loci were searched using NCBI Map Viewer build 36.2. To identify further paralagous genes, BLAST searches with putative protein sequences were conducted against the human genome, and putative positive targets were further characterised by molecular phylogenetics to resolve orthologous and paralagous relationships. Each of the genes from the putative human paralogons was cross-referenced by BLAST against the following NCBI genome assemblies; Mus musculus (build 37.1), Gallus gallus (build 2.1 ) and Danio rerio (Zv6) on NCBI map viewer. The Xenopus tropicalis (v4.1) and fugu rubripes (v4.0) genomes were searched at the Joint Genome Institute genome portal, and the Tetraodon nigroviridis (v8), Oryzias latipes (MEDAKA1) and Gasterosteus aculeatus (BROAD S1) genomes were searched via the Ensembl server. Putative target sequences were further analysed by molecular phylogenetics, to establish orthologous and paralogous relationships and to reaffirm our original classification of human sequences. For molecular phylogenetic analyses, protein sequences were first aligned, (with invertebrate outgroup sequences included where possible) using Clus-
talX and edited by eye [42]. Phylogenetic analyses were then carried out using maximum likelihood implemented by ClustalX [42], and by PHYML [43,44].

\section{Availability and requirements}

\section{JGI: http://genome.jgi-psf.org/Brafl1/Brafl1.home.html}

\section{Authors' contributions}

SD and KL conceived of the study, KW and FW performed the phylogenetic analyses, KW, FW, KL and SD equally contributed to the genomic searches, identification and characterisation of Lbx loci, and to the design of the manuscript. All authors read and approved the final manuscript.

\section{Additional material}

\section{Additional file 1}

Genomic arrangement of the Lbx paralogons.

Click here for file

[http://www.biomedcentral.com/content/supplementary/1471-

2148-8-171-S1.ppt]

\section{Additional file 2}

Description of gene families co-localising with Lbx/Tlx loci, and their phylogenetic analysis. Also included in this file are a list of abbreviations, the accession numbers of each sequence and a suggested new nomenclature for some of the genes.

Click here for file

[http://www.biomedcentral.com/content/supplementary/14712148-8-171-S2.pdf]

\section{Acknowledgements}

We are grateful to P. Holland, J. Postlethwait, P. Currie and S. Kuratani for inspiring discussions on Nk/Lbx gene evolution and to the reviewers for helpful comments on this manuscript. Funding for this study was provided 
by the EU Network of Excellence Myores to SD and a Royal Society University Research Fellowship to KEL. Both laboratories contributed equally to the work.

\section{References}

I. Jagla K, Bellard M, Frasch M: A cluster of Drosophila homeobox genes involved in mesoderm differentiation programs. Bioessays 200I, 23:125-133.

2. Pollard SL, Holland PW: Evidence for $\mathbf{1 4}$ homeobox gene clusters in human genome ancestry. Curr Biol 2000, 10:1059-1062.

3. Luke GN, Castro LF, McLay K, Bird C, Coulson A, Holland PW: Dispersal of NK homeobox gene clusters in amphioxus and humans. Proc Natl Acad Sci USA 2003, 100:5292-5295.

4. Holland PW, Booth HA, Bruford EA: Classification and nomenclature of all human homeobox genes. BMC Biol 2007, 5:47.

5. Larroux C, Fahey B, Degnan SM, Adamski M, Rokhsar DS, Degnan BM: The NK homeobox gene cluster predates the origin of Hox genes. Curr Biol 2007, I 7:706-7। 0 .

6. Schafer K, Braun T: Early specification of limb muscle precursor cells by the homeobox gene Lbxlh. Nat Genet 1999, 23:213-216.

7. Gross MK, Moran-Rivard L, Velasquez T, Nakatsu MN, Jagla K, Goulding M: LbxI is required for muscle precursor migration along a lateral pathway into the limb. Development 2000, 1 27:41 3-424.

8. Brohmann $\mathrm{H}$, Jagla $\mathrm{K}$, Birchmeier $\mathrm{C}$ : The role of Lbx I in migration of muscle precursor cells. Development 2000, 127:437-445.

9. Martin BL, Harland RM: A novel role for IbxI in Xenopus hypaxial myogenesis. Development 2006, 133:195-208.

10. Gross MK, Dottori M, Goulding M: LbxI specifies somatosensory association interneurons in the dorsal spinal cord. Neuron 2002, 34:535-549.

II. Muller T, Brohmann H, Pierani A, Heppenstall PA, Lewin GR, Jessell TM, Birchmeier C: The homeodomain factor Ibx I distinguishes two major programs of neuronal differentiation in the dorsal spinal cord. Neuron 2002, 34:55I-562.

12. Cheng L, Samad OA, Xu Y, Mizuguchi R, Luo P, Shirasawa S, Goulding $M, M a Q$ : LbxI and TIx3 are opposing switches in determining GABAergic versus glutamatergic transmitter phenotypes. Nat Neurosci 2005, 8: 1510-1515.

13. Kruger $M$, Schafer K, Braun T: The homeobox containing gene LbxI is required for correct dorsal-ventral patterning of the neural tube. J Neurochem 2002, 82:774-782.

14. Neyt C, Jagla K, Thisse C, Thisse B, Haines L, Currie PD: Evolutionary origins of vertebrate appendicular muscle. Nature 2000 408:82-86.

15. Dietrich S, Schubert FR, Healy C, Sharpe PT, Lumsden A: Specification of the hypaxial musculature. Development 1998 I 25:2235-2249.

16. Schubert FR, Dietrich S, Mootoosamy RC, Chapman SC, Lumsden A: LbxI marks a subset of interneurons in chick hindbrain and spinal cord. Mech Dev 200I, I0I:18I-I85.

17. De Graeve F, Jagla T, Daponte JP, Rickert C, Dastugue B, Urban J, Jagla $K$ : The ladybird homeobox genes are essential for the specification of a subpopulation of neural cells. Dev Biol 2004, 270: $122-134$.

18. Kanamoto T, Terada K, Yoshikawa H, Furukawa $\mathrm{T}$ : Cloning and expression pattern of Ibx3, a novel chick homeobox gene. Gene Expr Patterns 2006, 6:241-246.

19. Chen F, Liu KC, Epstein JA: Lbx2, a novel murine homeobox gene related to the Drosophila ladybird genes is expressed in the developing urogenital system, eye and brain. Mech Dev 1999, 84:181-184.

20. Holland PW, Garcia-Fernandez J, Williams NA, Sidow A: Gene duplications and the origins of vertebrate development. Dev Suppl 1994:125-133.

21. Taylor JS, Braasch I, Frickey T, Meyer A, Peer Y Van de: Genome duplication, a trait shared by $\mathbf{2 2 0 0 0}$ species of ray-finned fish. Genome Res 2003, 13:382-390.

22. Amores A, Force A, Yan YL, Joly L, Amemiya C, Fritz A, Ho RK, Langeland J, Prince V, Wang YL, et al.: Zebrafish hox clusters and vertebrate genome evolution. Science 1998, 282:17|I-17|4.

23. Wittbrodt J, Meyer A, Schartl M: More genes in fish? Bio-Essays 1998, 20:51|-515.
24. Wolfe K: Robustness-it's not where you think it is. Nat Genet 2000, 25:3-4.

25. Postlethwait $\mathrm{JH}$ : The zebrafish genome in context: ohnologs gone missing. J Exp Zoolog B Mol Dev Evol 2007, 308:563-577.

26. Taylor JS, Peer Y Van de, Braasch I, Meyer A: Comparative genomics provides evidence for an ancient genome duplication event in fish. Philos Trans R Soc Lond B Biol Sci 200I, 356:166I-1679.

27. Wotton KR, Shimeld SM: Comparative genomics of vertebrate Fox cluster loci. BMC Genomics 2006, 7:27I.

28. Mulley JF, Chiu CH, Holland PW: Breakup of a homeobox cluster after genome duplication in teleosts. Proc Natl Acad Sci USA 2006, 103:10369-10372.

29. Hoegg S, Meyer A: Phylogenomic analyses of KCNA gene clusters in vertebrates: why do gene clusters stay intact? $B M C$ Evol Biol 2007, 7:139.

30. Dehal $P$, Boore $\mathrm{JL}$ : Two rounds of whole genome duplication in the ancestral vertebrate. PLOS Biol 2005, 3:e314.

31. Lundin LG: Evolution of the vertebrate genome as reflected in paralogous chromosomal regions in man and the house mouse. Genomics 1993, 16:1-19.

32. Pebusque MJ, Coulier F, Birnbaum D, Pontarotti P: Ancient largescale genome duplications: phylogenetic and linkage analyses shed light on chordate genome evolution. Mol Biol Evol 1998, I5: I| 45-II59.

33. Gibson TJ, Spring J: Evidence in favour of ancient octaploidy in the vertebrate genome. Biochem Soc Trans 2000, 28:259-264.

34. Abi-Rached L, Gilles A, Shiina T, Pontarotti P, Inoko H: Evidence of en bloc duplication in vertebrate genomes. Nat Genet 2002, 3I:100-105.

35. Castro LF, Holland PW: Chromosomal mapping of ANTP class homeobox genes in amphioxus: piecing together ancestral genomes. Evol Dev 2003, 5:459-465.

36. Panopoulou G, Hennig S, Groth D, Krause A, Poustka AJ, Herwig R, Vingron $M$, Lehrach $\mathrm{H}$ : New evidence for genome-wide duplications at the origin of vertebrates using an amphioxus gene set and completed animal genomes. Genome Res 2003, 13:1056-1066.

37. Castro LF, Furlong RF, Holland PW: An antecedent of the MHClinked genomic region in amphioxus. Immunogenetics 2004, 55:782-784.

38. Vienne A, Rasmussen J, Abi-Rached L, Pontarotti P, Gilles A: Systematic phylogenomic evidence of en bloc duplication of the ancestral 8pII.2I-8p2I.3-like region. Mol Biol Evol 2003, 20: $1290-1298$.

39. Dear TN, Sanchez-Garcia I, Rabbitts TH: The HOXII gene encodes a DNA-binding nuclear transcription factor belonging to a distinct family of homeobox genes. Proc Natl Acad Sci USA 1993, 90:4431-4435.

40. Langenau DM, Palomero T, Kanki JP, Ferrando AA, Zhou Y, Zon LI, Look AT: Molecular cloning and developmental expression of TIx (Hox I I) genes in zebrafish (Danio rerio). Mech Dev 2002, I I 7:243-248.

4I. Logan C, Wingate RJ, McKay IJ, Lumsden A: TIx-I and TIx-3 homeobox gene expression in cranial sensory ganglia and hindbrain of the chick embryo: markers of patterned connectivity. J Neurosci 1998, 18:5389-5402.

42. Thompson JD, Gibson TJ, Plewniak F, Jeanmougin F, Higgins DG: The CLUSTAL_ $X$ windows interface: flexible strategies for multiple sequence alignment aided by quality analysis tools. Nucleic Acids Res 1997, 25:4876-4882.

43. Guindon S, Gascuel O: A simple, fast, and accurate algorithm to estimate large phylogenies by maximum likelihood. Syst Biol 2003, 52:696-704.

44. Guindon S, Lethiec F, Duroux P, Gascuel O: PHYML Online-a web server for fast maximum likelihood-based phylogenetic inference. Nucleic Acids Res 2005, 33:W557-559. 